\title{
Subcutaneous Sarcoidosis Mimicking Cellulitis
}

KYU YEOUN WON, MD, PhD, Department of Pathology, Kyung Hee University Hospital at Gangdong, Seoul, Republic of Korea; SO YOUNG PARK, MD, PhD, Department of Radiology, Kyung Hee University Hospital at Gangdong, Seoul, Republic of Korea; SANG-HOON LEE, MD, PhD, Division of Rheumatology, Department of Internal Medicine, Kyung Hee University Hospital at Gangdong, Seoul, Republic of Korea. Address correspondence to Dr. S.Y. Park, Department of Radiology, Kyung Hee University Hospital at Gangdong, 892, Dongnam-ro, Gangdong-gu, Seoul 134-727, Republic of Korea. E-mail: balgundol@hanmail.net. J Rheumatol 2016;43:674-5; doi:10.3899/jrheum.151030

Sarcoidosis is a systemic disorder of unknown etiology characterized by noncaseating granulomas ${ }^{1}$. The lesion can have a reticular pattern similar to cellulitis.

A 54-year-old woman with a 5-month history of focal swelling in the right medial thigh visited our hospital. The patient was treated under the assumption of cellulitis at an outpatient clinic. However, the mass-like lesion remained unchanged. Erythrocyte sedimentation rate was slightly elevated $(27 \mathrm{~mm} / \mathrm{h})$ and other laboratory analyses yielded normal results. Ultrasound (US) revealed a focal ill-defined subcutaneous (SC) lesion with a reticular pattern, similar to cellulitis (Figure 1). There was no change in the lesion upon followup US 2 weeks later. Magnetic resonance imaging (MRI) of the right thigh showed an ill-defined lesion with a nodule (arrowhead) as well as a reticular pattern (arrow; Figure 2). US-guided biopsy was performed. Histological analysis revealed several foci of noncaseous granulomas in the SC layer. The granulomas were composed of aggregations of epithelioid histiocytes and multinucleated giant cells. Surrounding lymphoplasmacytic infiltration was noted (Figure 3). No acid-fast bacilli were found and no Mycobacterium tuberculosis was detected by PCR. Foreign material and fungal organism were not identified. A histopathologic diagnosis of sarcoidosis was made. Chest

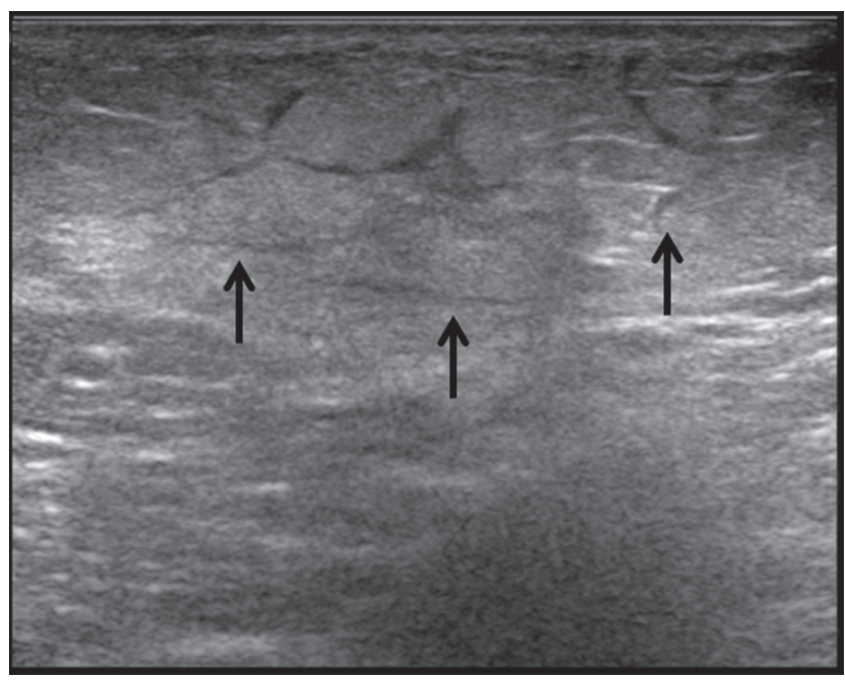

Figure 1. A longitudinal ultrasound scan of the right medial thigh showed an ill-defined lesion with a low echoic reticular pattern (arrows).

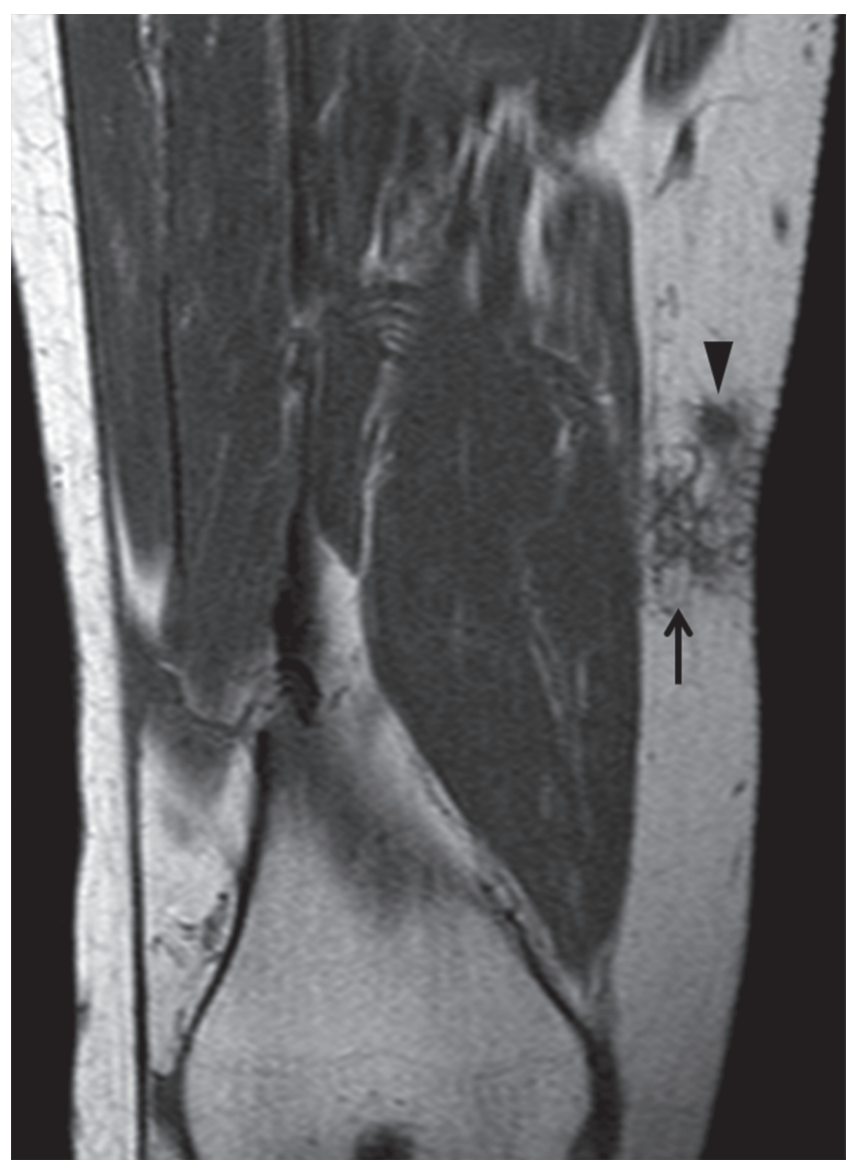

Figure 2. Coronal T1-weighted magnetic resonance imaging of the right thigh demonstrated an ill-defined lesion with a reticular pattern (arrow) and a nodular area (arrowhead).

computed tomography showed that lungs and mediastinum were normal. The patient was treated with oral methylprednisolone and cyclosporine, and the mass-like lesion disappeared.

The US and MRI findings of SC sarcoidosis include illdefined lesions with reticular pattern, nodular or mass-like areas, or diffuse infiltrates in the SC layer ${ }^{2,3,4}$. When an SC lesion showing the cellulitis-like imaging findings is not associated with erythema, warmth, and pain, SC sarcoidosis should be considered. 


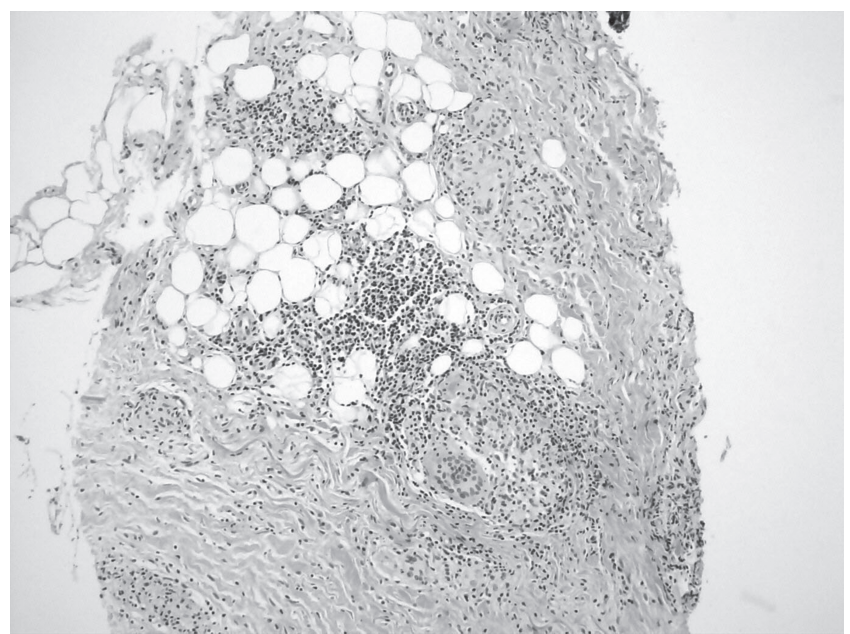

Figure 3. Microscopically, the section shows several foci of small noncaseous granulomas in the subcutaneous layer. The granulomas were composed of aggregations of epithelioid histiocytes and multinucleated giant cells $(H \& E$ stain, original magnification $\times 100)$.

\section{REFERENCES}

1. Heinle R, Chang C. Diagnostic criteria for sarcoidosis. Autoimmun Rev 2014;13:383-7.

2. Vardhanabhuti V, Pang CL, Ninan T, Adams WM, Raju V, Suresh P. Sarcoidosis - the greatest mimic. Semin Ultrasound CT MR 2014;35:215-24.

3. Bosnić D, Baresić M, Bagatin D, Ilić I. Subcutaneous sarcoidosis of the face. Intern Med 2010;49:589-92.

4. Chen HH, Chen YM, Lan HH, Lee CH, Chen DY. Sonographic appearance of subcutaneous sarcoidosis. J Ultrasound Med 2009;28:813-6. 